\title{
Evaluation of BOLD effects in the rat cortex
}

2 Nathalie Just

3

4 Corresponding author: Nathalie Just, $\mathrm{PhD}, \mathrm{HDR}$

5 INRAe Centre Val de Loire

6 UMR Physiologie de la Reproduction et des Comportements

7 Equipe Néogenèse Hypothalamique, Rôles

8 Variations et perturbations endocrines - NHyRVana

937380 Nouzilly

10 France

11

12 E-Mail address: nathalie.just18@gmail.com

\section{Running head : Accuracy of ${ }^{\mathbf{1}} \mathbf{H}$-fMRS in rodents}




\section{Abstract}

2 Purpose: This study aimed to characterize Blood oxygen level-dependent (BOLD) effects in ${ }^{1} \mathrm{H}-\mathrm{MR}$

3 spectra obtained during optogenetic activation of the rat forelimb cortex for the correction and

4 estimation of accurate metabolite concentration changes.

5 Methods : $\mathrm{T}_{2}{ }^{*}$-induced effects were characterized by linewidth changes and amplitude changes of

6 water, NAA and tCr spectral peaks during the stimulation paradigm. Spectral linewidth-matching

7 procedures were used to correct for the line-narrowing effect induced by BOLD. For an increased

8 understanding of spectroscopic BOLD effects and the optimized way to correct them, a $1 \mathrm{~Hz}$ line-

9 narrowing effect was also simulated on mouseproton MR spectrum

$10 \quad{ }^{1} \mathrm{H}$-fMRS data acquired using STEAM acquisitions at $9.4 \mathrm{~T}$ in rats $(\mathrm{n}=8)$ upon optogenetic stimulation

11 of the primary somatosensory cortex were used. Data were analyzed with MATLAB routines and

12 LCModel. Uncorrected and corrected ${ }^{1} \mathrm{H}-\mathrm{MR}$ spectra of simulated and in-vivo data were quantified

13 and compared. BOLD-corrected difference spectra were also calculated and analyzed.

14 Results: Significant mean increases in water and NAA peak heights $(+1.1 \%$ and $+4.5 \%$, respectively)

15 were found accompanied by decreased linewidths $(-0.5 \mathrm{~Hz}$ and $-2.8 \%)$ upon optogenetic stimulation.

16 These estimates were used for further definition of an accurate line-broadening factor (lb). Usage of an

17 erroneous $\mathrm{lb}$ introduced false-positive errors in metabolite concentration change estimates thereby

18 altering the specificity of findings. Using different water scalings within LCModel, the water and

19 metabolite BOLD contributions were separated.

20 Conclusion : The linewidth-matching procedure using a precise $\mathrm{lb}$ factor remains the most performant

21 approach for the accurate quantification of small $( \pm 0.3 \mu \mathrm{mol} / \mathrm{g})$ metabolic changes in ${ }^{1} \mathrm{H}-\mathrm{fMRS}$

22 studies. A simple and preliminary compartmentation of BOLD effects was proposed, which will 23 require validation.

24

25 Keywords : ${ }^{1} \mathrm{H}-\mathrm{fMRS}$, rats, BOLD effects, metabolite concentrations, line broadening 
2 The investigation of neurochemical changes during brain activity is important for the 3 improved interpretation of neurovascular coupling mechanisms in the healthy and diseased

4 brain [1,2]. In this context, proton functional MR spectroscopy $\left({ }^{1} \mathrm{H}-\mathrm{fMRS}\right)$ represents a

5 technique of particular interest when investigating the underpinnings of functional MR

6 imaging signals $[1,2]$ both in the human brain and in animal models. ${ }^{1} \mathrm{H}$-fMRS techniques

7 generate more and more interest allowing for the reproducible assessment of activity-induced

8 changes of important neurotransmitters concentrations such as glutamate (Glu) and g-

9 aminobutyric acid (GABA) as well as energy metabolites such as Glucose (Glc) and Lactate

10 (Lac) [1, 3]. Interestingly, absolute functional changes of these metabolite concentrations

11 demonstrated to be rather small $(\sim 0.2 \mu \mathrm{mol} / \mathrm{g})$ in humans $[4,5,6]$. The absolute quantification

12 of these changes was performed following the correction of $\mathrm{T}_{2}$-induced effects [7].

$\mathrm{T}_{2} *$-induced effects (or BOLD effects) induce a narrowing of spectral peak linewidths as well

14 as $\mathrm{T}_{2}$ changes, as a result of the increased oxygenation within the activated region of interest.

15 These decreases were mainly observed on water, N-Acetyl-Aspartate (NAA) and total

16 Creatine $(\mathrm{tCr}=\mathrm{PCr}+\mathrm{Cr})$ singlets and represented only $2 \%$ change during visual stimulation

17 of the human visual cortex. They were more easily observable as relative increases in metabolite peak height of around 3\% [7]. Although the change in metabolite concentration

19 due to $\mathrm{T}_{2} *$-induced effects is low (less than $1 \%$ ), if left uncorrected, it may lead to a high

20 degree of false-discovery rate [8]. Errors may become particularly important when absolute 21 changes as low as $0.2 \mu \mathrm{mol} / \mathrm{g}$ are expected. For an accurate estimation of metabolite 22 concentration changes, Mangia et al. $[4,5]$ proposed to correct for the BOLD effects by line 23 broadening the population-averaged stimulated spectrum to match the linewidth of the 24 corresponding population-averaged REST spectrum. The corrected stimulated and REST 25 spectra were subsequently subtracted resulting in BOLD-free difference spectrum. Positive 
1 Glu and Lac peaks were visually identified and subsequently quantified using a simulated

2 difference basis set within LCModel [9]. This procedure demonstrated reproducible outcome

3 within the human primary visual cortex at a high magnetic field owing to the large SNR

4 available $[4,5,6,8,10,11,12]$.

5 In rodents, ${ }^{1} \mathrm{H}$-fMRS studies remain challenging and have shown lower quantitative

6 reproducibility $[13,14,15]$. The methodology for obtaining accurate estimates of metabolite

7 concentration changes during brain activation remains difficult to replicate in animal models

8 with a voxel of interest more than 500 times smaller than in the human brain $[13,14,15,16]$.

9 The advent of revolutionary techniques such as optogenetics and chemogenetics that can be

10 coupled to ${ }^{1} \mathrm{H}$-fMRS [16] for the specific stimulation of excitatory cell populations could

11 increase its potential if accurate quantification of metabolic concentration changes can be

12 achieved.

13 While the difference-spectrum procedure is amplitude-based and pretty straightforward, the

14 line broadening procedure followed by LCModel quantification is ambiguous because areas

15 under the spectral peaks are calculated. On that account, peak line broadening should not

16 affect metabolite quantification. This is, however, not the case. Such errors in metabolite

17 fitting models using linear combination modelling algorithms (such as LCModel) have been

18 scarcely reported in the literature but interest is growing as more accuracy of metabolite

19 concentration estimates and more standardization across processing methods are needed [17,

20 18]. Besides, these algorithms do not account for the change of the apparent $T_{2}$ value.

21 In ${ }^{1} \mathrm{H}$-fMRS studies at high magnetic field strength, it is of paramount importance to remove

22 BOLD effects or at least to determine how they affect neurochemical profiles. In human

23 studies at $7 \mathrm{~T}$ with high SNR levels, corrections were applied and deemed adequate as they

24 compared well with results from difference spectrum procedures [8]. How were these

25 corrections optimised ? How was it decided they were suitable and how can they be 
1 appropriately applied to data with poorer SNR ?

2 In the present paper, BOLD effects and their correction were examined with simulated data

3 and on in-vivo proton MR spectra acquired in rats during photo-stimulation of the primary

4 somatosensory forelimb cortex (S1FL). The aim of this preliminary work was to investigate

5 the robustness of the line-matching procedure to correct for BOLD effects and the validity of

6 this corretion within LCModel. In addition, a simple method to better isolate and separate

7 BOLD contributions from metabolite concentrations was proposed.

\section{2. METHODS}

\section{$9 \quad$ 2.1.1 Simulations}

10 A highly resolved ${ }^{1} \mathrm{H}-\mathrm{MR}$ spectrum acquired at 9.4T with a STEAM sequence and a mouse 11 cryoprobe (Bruker Biospin GmbH, Ettlingen, Germany) in the thalamus of a healthy mouse 12 was used to represent a stimulated ${ }^{1} \mathrm{H}$ MR spectrum (STIM). This ${ }^{1} \mathrm{H}$ MR spectrum was line13 broadened $(\mathrm{lb}=1 \mathrm{~Hz})$ so that the difference in spectral amplitude of NAA represented a $2 \%$ 14 change according to literature values and represented the REST ${ }^{1} \mathrm{H}$ MR spectrum (REST). 15 White noise was added to compensate for the smoothing effect. The difference in NAA 16 amplitude was assumed to represent the line narrowing effect induced by the BOLD effects.

17 Metabolite concentrations were quantified using LCModel using two reference standards : (a)

18 the internal water signal from the unsuppressed water scan acquired in the same mouse, which 19 was assumed to represent the BOLD contaminated water signal (for clarity purpose, it was 20 called waterBOLD) and (b) the internal water signal from the unsuppressed water scan 21 acquired in the same mouse and line broadened (to represent a $2 \%$ signal decrease compared 22 to waterBOLD), which was assumed to represent the water signal at REST (waterREST).

\section{$23 \quad$ 2.1.2 Animals and surgery}

24 All experiments were performed according to the German Tierschutzgesetz and were 
1 approved by local authorities (Landesamt für Natur, Umwelt und Verbraucherschutz

2 Nordrhein-Westfalen, Germany). 8 Female Fisher rats (F344) were considered in the present

3 study. Each of them underwent two craniotomies above the primary somatosensory forelimb

4 cortex (S1FL) for the injection of a viral construct [19] and the optic fibre (OF) implantation

5 on the day of MR acquisitions. An OF of $200 \mu \mathrm{m}$ in diameter was inserted only superficially

6 above S1FL at a depth of $100 \mu \mathrm{m}$ and glued to the skull. Each rat was intubated under

7 isoflurane anaesthesia in oxygen (2-2.5\%) and one of the caudal veins was catheterized for

8 infusing pancuronium bromide $(1 \mathrm{mg} / \mathrm{ml} \mathrm{BW})$ during fMRI-fMRS acquisitions. The rat skull

9 was covered with warm agarose gel (1\%) to further prevent air-tissue susceptibility artefacts.

10 After fixing the rat head using ear and bite bars, the rat was positioned in a dedicated cradle.

11 Respiration was monitored throughout the entire duration of the experiments as well as end-

12 tidal $\mathrm{CO}_{2}$ measurements performed through a capnometer (CapStar-100 $\mathrm{CO}_{2}$ Analyzer, CWE

13 Inc., Ardmore, PA, USA) and maintained between 2.8 and 3.5\%. The temperature was

14 measured through a rectal probe and maintained at $37 \pm 1^{\circ} \mathrm{C}$ via water tubing linked to a

15 temperature retro-controlled bath.

\section{2.2. Magnetic Resonance Imaging and spectroscopic experiments}

\section{$17 \quad 2.1$ BOLD-fMRI}

18 All MR experiments were performed at 9.4T (Biospec 94/20, Bruker Biospin GmbH,

19 Ettlingen, Germany) in a small animal MR scanner equipped with $0.7 \mathrm{~m} / \mathrm{T}$ gradients using a

20 20mm-single loop surface coil for the reception and a 90-mm volume coil for transmission

21 (Rapid Biomedical gmbH, Rimpar, Germany). After the acquisition of pilot and anatomical

22 images, anaesthesia was switched to medetomidine (Domitor, Pfizer, Orion Corporation,

23 Espoo, Finland) $(0.04 \mathrm{mg} / \mathrm{kg}$ (bolus) $+0.05 \mathrm{mg} / \mathrm{kg} / \mathrm{hr}$ (subcutaneous infusion)) and isoflurane

24 was discontinued. The first single-shot gradient-echo echo-planar imaging (TR/TE=

$251000 / 18 \mathrm{~ms} ;$ FOV=28 x 26mm; Matrix $=80 \times 80 ;$ Bandwidth=200-300 kHz; TH=0.8mm; 16 
1 slices; 600 images) took place at least 1 hour after the start of medetomidine infusion and

2 after whole-brain shimming using MAPSHIM. Laser pulses were delivered successively using

3 in-house developed programs allowing triggering and repetition of a 10sOFF-10sON-10sOFF

4 paradigm (pulse frequency of $9 \mathrm{~Hz}$; pulse duration of $10 \mathrm{~ms}$ ). The power calibrated green laser

5 light $(552 \mathrm{~nm})$ was delivered ensuring a mean power intensity below $22 \mathrm{~mW} / \mathrm{mm}^{2}$ at the tip of

6 the OF to avoid heat effects [20]. BOLD-fMRI images were processed using SPM12 as

7 described earlier ${ }^{13}$.

\section{$8 \quad$ 2.2.2 functional Magnetic Resonance Spectroscopy (fMRS)}

9 Localized functional proton MR spectroscopy was conducted using a STEAM sequence (TR= $104 \mathrm{~s} ; \mathrm{TE}=2.6 \mathrm{~ms} ; \mathrm{TM}=10 \mathrm{~ms} \mathrm{SW}=4960 \mathrm{~Hz} ; 4096$ points). An $8 \mu \mathrm{l}$ VOI was positioned onto 11 the anatomical $\mathrm{T}_{2}$-weighted RARE images coregistered to the GRE-EPI images ensuring that 12 most of the VOI encompassed the BOLD responses while lipid contamination from the skull 13 was minimized. The water signal was suppressed using the VAPOR module [21] and three 14 modules of outer volume saturation (OVS) were interleaved with the water suppression 15 pulses. A $3 \times 3 \times 3 \mathrm{~mm}^{3}$ voxel was placed over the rat cortex and used for shimming down to a water linewidth of $15 \mathrm{~Hz}$ using first and second-order FASTMAP shimming. A 2.5 min OFF-

$175 \mathrm{~min}$ ON-2.5min OFF paradigm was used representing 45 minutes of acquisition (650 FIDs) and repeated 4 times per rat. Unsuppressed water spectra were also acquired using the same 19 sequence and paradigm to provide reference water peaks for eddy current correction and 20 further metabolite quantification. STEAM ${ }^{1} \mathrm{H}$ MR spectra were reconstructed and 21 preprocessed using in-house written MATLAB routines. For each rat, raw ${ }^{1} \mathrm{H}$ spectra were 22 corrected for frequency drift and FIDs were summed across stimulation and rest periods, 23 respectively, and transferred to a SUN station for LCModel analysis [9] using a basis set 24 provided by Steven Provencher containing a simulated set of macromolecules as well as the 25 following metabolites: Scyllo-Inositol: Scyllo; Alanine: Ala; Aspartate: Asp; Glycero- 
1 phosphocholine: GPC; Phosphocholine: PCh; Creatine: Cr; Phosphocreatine: PCr; $\gamma$ -

2 aminobutyric acid: GABA: Glucose: Glc; Glutamine: Gln; Glutamate: Glu: Glutathione:

3 GSH; myo-inositol: Ins; Lactate: Lac; N-acetyl aspartate: NAA; N-acetylaspartyl-glutamic

4 acid: NAAG; Phosphatidylethanolamine: PE; Taurine: tau. $\mathrm{tCr}$ : total creatine $(\mathrm{PCr}+\mathrm{Cr})$; Glx:

5 Glu + Gln; Absolute metabolite concentrations were obtained using unsuppressed water

6 signals as an internal reference assuming a brain water content of $80 \%$. The Cramer-Rao

7 Lower bounds (CRLB) were used as a reliability measure of the metabolite concentration

8 estimates. Only metabolites CRLB below $15 \%$ were kept for further analysis.

\subsection{Data analysis}

\subsubsection{Estimation of $\mathrm{T}_{2}{ }^{*}$-induced effects on NAA and $\mathrm{tCr}$ spectral peaks}

A moving average was used to calculate NAA and $\mathrm{tCr}$ signal time courses. Each metabolite concentration-time point was obtained by summing four consecutive blocks of eight FIDs. The procedure was applied to each of the four functional MR spectroscopic acquisitions obtained during the delivery of green laser paradigms. Time courses were then averaged across the animal population. The temporal resolution was $42 \mathrm{~s}$. Unsuppressed water signal time courses were obtained using a 4 s temporal resolution for each rat and averaged over the population of rats.

\subsubsection{Estimation of metabolite concentration changes}

After phasing and correction for B0 shifts, 1200 FIDs were summed per animal and then across 8 rats for the 5-min rest periods and the 5-min stimulation periods resulting in a STIM spectrum and a REST spectrum. STIM and REST spectra were then individually fitted using LCModel. Metabolite concentrations were obtained using either the temporally averaged unsuppressed water peak obtained during resting periods or temporally averaged unsuppressed water peak obtained during stimulation periods. The latter procedure was termed the WATERBOLD approach as described in the theory section. 
1 STIM and REST spectra were also subtracted from each other. BOLD-corrected STIM

2 spectra (STIMC) were obtained using a line-broadening factor lb. lbs were increased in steps

3 of $0.2 \mathrm{~Hz}$ and applied to the NAA peak of the averaged stimulated spectrum to match the

4 linewidth of the NAA peak of the averaged REST spectrum. The lb value that best minimized

5 the residual NAA difference peak between STIM and REST was used. STIMC spectra were

$6 \quad$ LCModel fitted.

7 All procedures were performed using an in-house written Matlab routine. BOLD-corrected

8 difference spectra also obtained (STIMC-REST) and fitted using LCModel after simulation

9 of a basis set containing positive lactate and glutamate changes and negative aspartate and

$10 \quad$ Glucose changes.

\section{$11 \quad$ 2.4. Statistics}

12 A Shapiro-Wilk test was performed for all the metabolite concentrations quantified during

13 REST and stimulation conditions demonstrating normality of all the distributions with a p-

14 value above 0.05. Repeated measures One-way ANOVA tests followed by Bonferroni posthoc

15 tests (11 metabolites) to correct for multiple comparisons were used to compare changes in

16 metabolite concentrations. Results were presented as mean \pm CRLB. A p-value under 0.05

17 was considered significant.

18 Averaged per cent changes in signal time courses between REST and stimulation periods

19 were compared using a paired student t-test. A p-value under 0.05 was considered significant.

20 Results were presented as mean \pm standard deviation.

\section{$21 \quad$ 3. RESULTS}

\subsection{Simulations of BOLD responses obtained with fMRI and fMRS}

\subsubsection{Comparison of BOLD responses}

24 For a better understanding of the relationship between $\mathrm{T}_{2} *$ and spectral peak linewidths during localized brain activation, equations relating to these entities were used. 
1 The full width at half maximum (FWHM) of a spectral peak obeys the following equation:

$2 \quad \mathrm{FWHM}=1 / \pi \mathrm{T}_{2} *(1)$

3 Changes in $R_{2} *$ due to focal cerebral activity can be expressed as : $\Delta R_{2} *=-1 / T_{E} * \ln (1+B O L D)$

5 allowing the change in linewidth $(\Delta \mathrm{lw})$ occasioned by the BOLD effects to be expressed as:

$6 \Delta \mathrm{FWHM}=1 / \pi \Delta \mathrm{T}_{2} *=-1 / \pi \mathrm{T}_{\mathrm{E}} * \ln (1+\mathrm{BOLD})(3)$

7 These equations were used to model and compare «BOLD-fMRI» and «BOLD-fMRS» 8 responses on water.

9 As the magnetic field strength increases, the $\mathrm{T}_{2} *$ relaxation time decreases significantly [22].

10 Upon BOLD activation of the human motor cortex, changes in relaxation rate $\Delta \mathrm{R}_{2} *$ increased

11 linearly as a function of field strength [23]. A higher spectral peak linewidth change due to

12 activation should therefore be expected at higher magnetic field strength for a same TE. The

13 assessment of $\mathrm{T}_{2}{ }^{*}$-induced effects due to hyperoxygenation of blood should be easier at high

14 magnetic field strength $(>7 \mathrm{~T})$.

15 Water linewidth changes $(\Delta \mathrm{lw})$ for $\mathrm{TE}=3,18,26 \mathrm{~ms}$, chosen from previous ${ }^{1} \mathrm{H}$-fMRS studies, 16 were calculated with Eq. (3) (Fig. 1). $\Delta$ lw changes evolved linearly with BOLD changes. At

$17 \mathrm{TE}=3 \mathrm{~ms}$, changes were elevated compared to those obtained at $\mathrm{TE}=18 \mathrm{~ms}(9.4 \mathrm{~T},[16]$ or

$18 \mathrm{TE}=26 \mathrm{~ms}(7 \mathrm{~T},[4,5])$.

19 For $\mathrm{BOLD}=2 \% \Delta \mathrm{lw}=-2.1 \mathrm{~Hz}(\mathrm{TE}=3 \mathrm{~ms}) ; \Delta \mathrm{lw}=-0.35 \mathrm{~Hz}(\mathrm{TE}=18 \mathrm{~ms}) ; \Delta \mathrm{lw}=-0.24 \mathrm{~Hz}$ $20 \quad$ (TE=26 ms); 
2

3

4

5

1,00
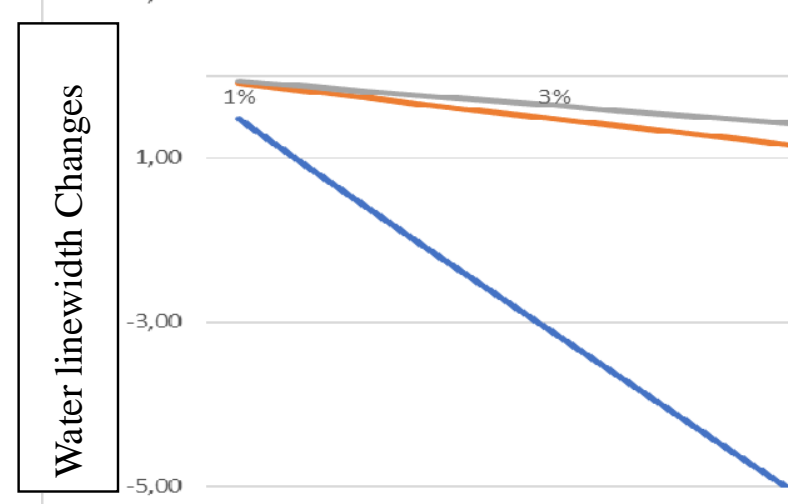

BOLD (\%)

Iw1 (TE=18ms)

Iw2 (TE=3ms) $\quad$ Iw $3(T E=26 \mathrm{~ms})$

Figure 1 Water linewidth changes versus BOLD-fMRI for different TEs using equations (1) and (3)

However for TE $=3 \mathrm{~ms}$, the BOLD effects measured with $\mathrm{fMRI}$ is negligible. For $\Delta \mathrm{T}_{2} *=1.5 \mathrm{~s}$ (arbitrarily chosen), BOLD $=0.47 \%$ for $\mathrm{TE}=3 \mathrm{~ms}$ when $\mathrm{BOLD}=2.9 \%$ for $\mathrm{TE}=18 \mathrm{~ms}$. This change corresponds to a linewidth change of $-0.5 \mathrm{~Hz}$ and $\mathrm{BOLD}=0.5 \%$ for $\mathrm{TE}=3 \mathrm{~ms}$ and $\mathrm{BOLD}=3 \%$ for $\mathrm{TE}=18 \mathrm{~ms}$. These values correspond well to linewidth changes quantified in vivo on NAA and Cr spectral lines [10,24].

\subsubsection{Quantification of metabolite concentrations affected by simulated BOLD effects}

To illustrate errors induced by the BOLD effects and an erroneous correction of this effect on metabolite quantification, a $2 \%$ BOLD effect was simulated that induced a $1 \mathrm{~Hz}$ decrease of the NAA spectral peak linewidth (Fig 2). Metabolite concentrations were quantified using LCModel [9]. In this example, no metabolic concentration changes occurred. The ground truth metabolic concentrations were obtained by LCModel adjustment of the non-stimulated spectrum (black spectrum). 

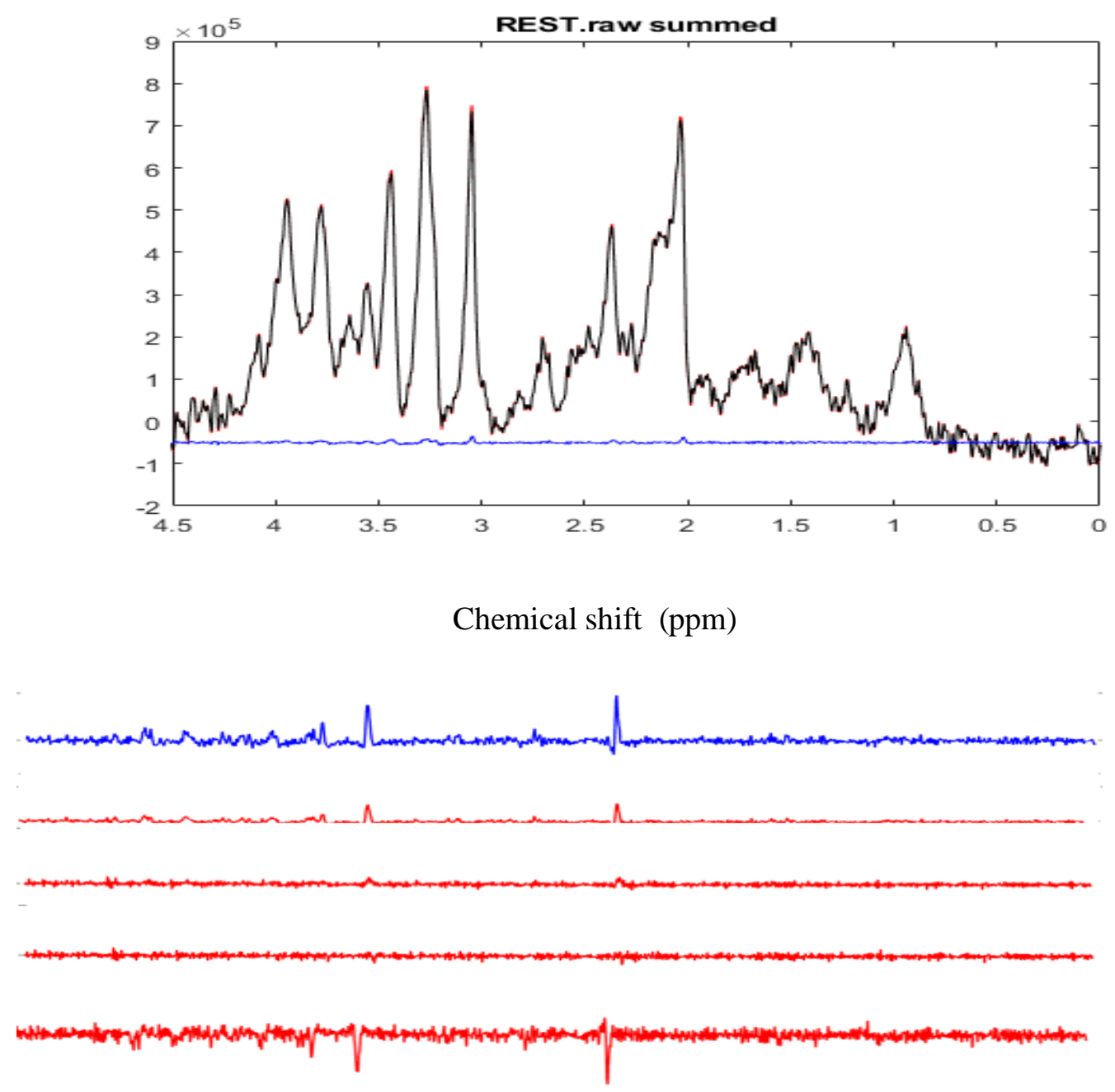

$1 \mathrm{~b}=1 \mathrm{~Hz}$

$1 \mathbf{b}=2 \mathrm{~Hz}$

Figure 2 : Simulation of BOLD effects : A. A highly spectrally resolved ${ }^{1}$ H-MR spectrum acquired at 9.4T in the mouse thalamus with a cryoprobe (red) served as a stimulated spectrum (STIM) and was exponentially line broadened by $1 \mathrm{~Hz}$ to represent a $2 \%$ amplitude decrease of the NAA peak in the resting spectrum (REST, black). The $2 \%$ difference represents the simulated BOLD effect as a result of $\mathrm{T}_{2}{ }^{*}$-induced effects. BOLD effects can also be seen as residual positive peaks in the difference spectrum (blue; STIM-REST) B. C. The REST spectrum was subtracted from the STIM spectrum demonstrating residual positive peaks due to the simulated BOLD effect. As the line-broadening factor lb applied to the STIM spectrum increased from $0.2 \mathrm{~Hz}$ to $1 \mathrm{~Hz}$, BOLD residuals gradually disappeared while negative residuals grew up for $\mathrm{lb}$ values above $1 \mathrm{~Hz}$ 
1 The correction for BOLD effects consists of the application of a line broadening factor, which

2 must be at least equal to the decrease of the spectral linewidth induced by focal cerebral

3 activity so that stimulated and non-stimulated spectral linewidths are equivalent. This

4 correction can be applied to the water peak [7] but most ${ }^{1} \mathrm{H}$-fMRS studies report it on N-

5 acetyl-aspartate (NAA) and total Creatine $(\mathrm{PCr}+\mathrm{Cr})$ peaks $[4,5,6,8,13]$. To observe

6 changes induced by the BOLD effects, MR spectra obtained during stimulation and REST are

7 subtracted. Residuals can be visually observed and correspond to the BOLD effects (Fig. 2).

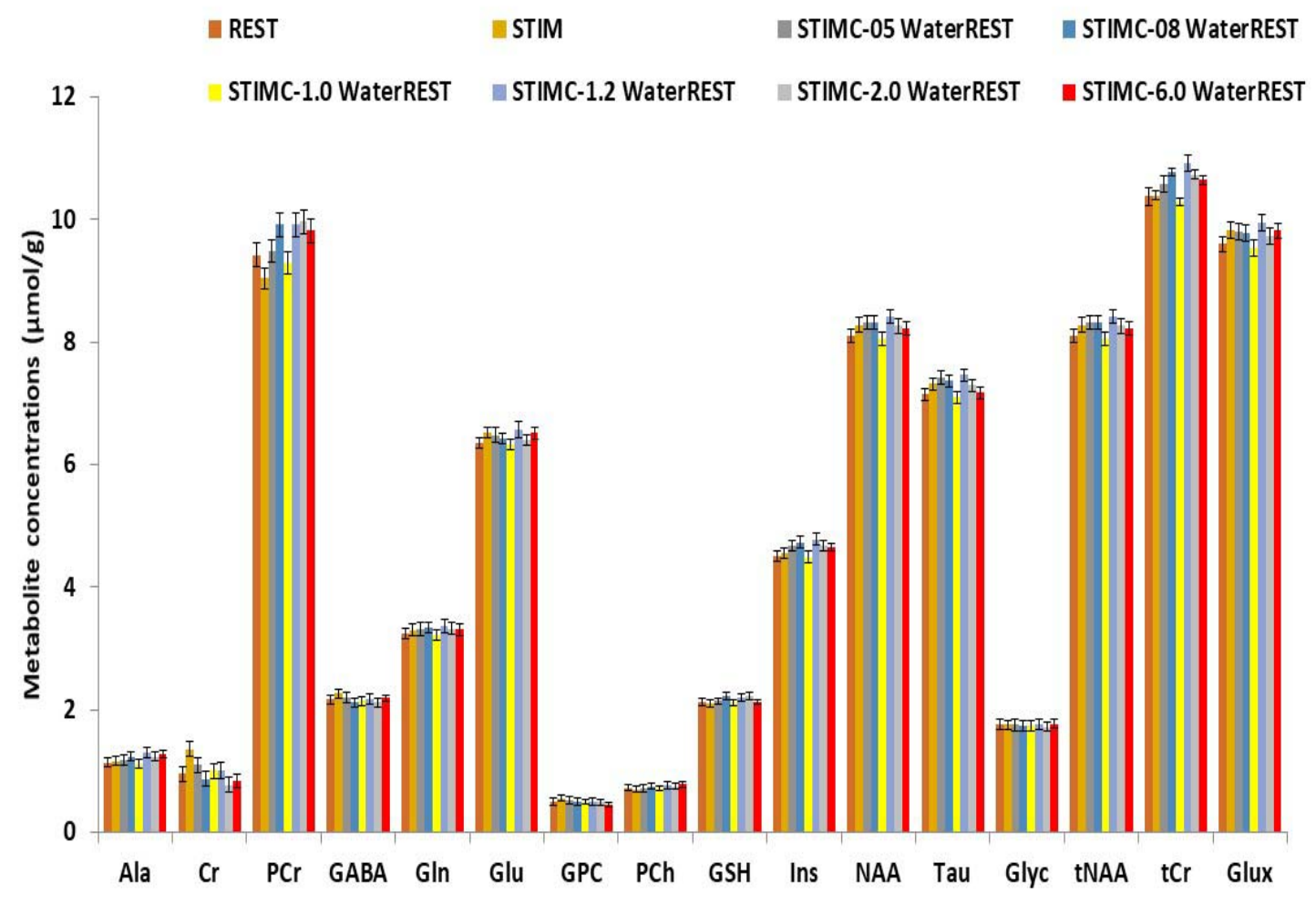

9 Figure 3: Comparison of neurochemical profiles. REST represents the ground truth for metabolic the simulated STIM spectrum. The correction that best approached the ground truth was for $l b=1 \mathrm{~Hz}$.

12 


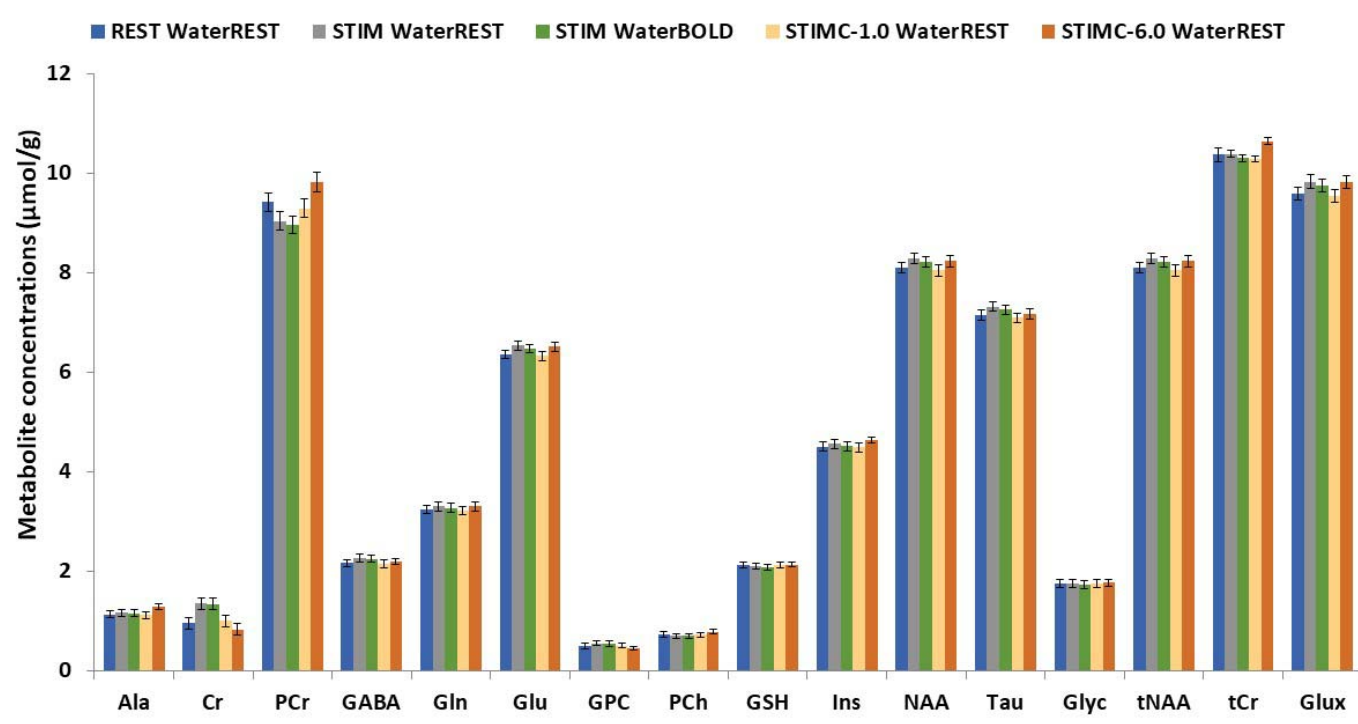

Figure 4:Comparison between the REST, STIM, STIMC and waterBOLD neurochemical profiles. STIMC is the neurochemical profile of the line-broadened STIM spectrum with $l b=1 \mathrm{~Hz}$. waterBOLD is the neurochemical profile of the STIM spectrum scaled with the stimulated water peak.

The corrected neurochemical profiles of the spectra depicted in Fig. 2 are displayed in Fig 3.

7 REST represents the neurochemical profile for «true» metabolite concentrations while

8 STIMC represents the corrected neurochemical profile for which a $1 \mathrm{~Hz}$ line broadening

9 correction was applied. This correction represents the optimum correction for BOLD effects

10 since metabolite concentration differences were minimized. The line broadening correction is

11 the most effective when it is the closest to the linewidth reduction induced by the BOLD

12 effects. The error relative to REST remained below $1 \%$ for $\mathrm{lb}=1 \mathrm{~Hz}$ for all the metabolites.

13 The error rose to $4 \%$ for $\mathrm{lb}=6 \mathrm{~Hz}$ for $\mathrm{PCr}$ and Ins and was of the same order of magnitude as

14 the error induced by the non-corrected BOLD effects $(\mathrm{lb}=0 \mathrm{~Hz})$.

In Fig 4, STIM represents the uncorrected BOLD effect neurochemical profile. The

16 quantification was performed with waterREST, the unsuppressed water spectrum acquired

17 before the REST spectrum. BOLD effects increased Glu concentrations by $2.6 \%$ and NAA

18 concentrations by $2.2 \%$. «waterBOLD» represents the neurochemical profile acquired during

19 stimulation but quantified using the simulated unsuppressed water peak with BOLD effects. 
1 Table 1 compares the Glu, NAA and $\mathrm{tCr}$ concentrations quantified with LCModel for the

2 different cases. When using waterBOLD for quantification, the BOLD effects increased Glu

3 concentrations by $1.8 \%$ and NAA concentrations by $1.4 \%$. The error induced by BOLD

4 effects, $\mathrm{BOLD}_{\text {water, }}$, on the water was calculated as the metabolite concentration difference

5 between STIM and waterBOLD and was approximately $0.8 \%$ for all metabolites. The error

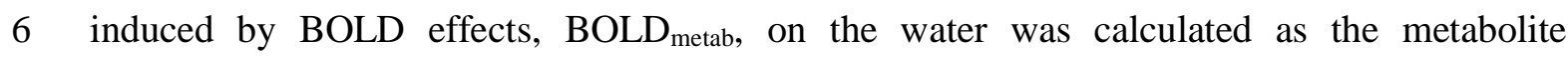

7 concentration difference between STIM and STIMC. Interestingly, BOLD effect-induced

8 errors were cumulative: $\mathrm{BOLD}_{\mathrm{STIM}}=\mathrm{BOLD}_{\text {metab }}+\mathrm{BOLD}_{\text {water }}$. Therefore, it is postulated that

9 estimates of metabolite concentrations with waterBOLD are affected by BOLD on

10 metabolites only.

11 Table 1 LCModel quantification of Glu, NAA and tCr $( \pm$ CRLB $)$ for simulated REST

and STIM spectra and STIM scaled with waterBOLD and the line-broadened STIM

spectrum (STIMC lb $=\mathbf{1} \mathrm{Hz})$

\begin{tabular}{|lllll|}
\hline & REST & STIM & waterBOLD & STIMC \\
Glu $(\boldsymbol{\mu m o l} / \mathbf{g})$ & $6.36 \pm 0.1$ & $6.5 \pm 0.1$ & $6.48 \pm 0.1$ & $6.32 \pm 0.1$ \\
$\mathbf{N A A}(\boldsymbol{\mu \mathrm { mol }} / \mathbf{g})$ & $8.11 \pm 0.1$ & $8.3 \pm 0.1$ & $8.22 \pm 0.1$ & $8.05 \pm 0.1$ \\
$\mathbf{t C r}(\boldsymbol{\mu \mathrm { mol }} / \mathbf{g})$ & $10.38 \pm 0.1$ & $10.4 \pm 0.1$ & $10.31 \pm 0.1$ & $10.29 \pm 0.1$ \\
\hline
\end{tabular}

\section{2 in-vivo characterization of BOLD effects}

16 In the rat S1FL, optogenetic stimulations resulted in an average BOLD response measured

17 with fMRI of $2.4 \pm 1.3 \%$ (Fig.5 A). A typical T-value BOLD map overlaid onto an anatomical

18 image is shown (Fig. 5A) as well as a typical BOLD timecourse following a 10-minute (10s

19 ON- 20s OFF) stimulation paradigm (Fig. 5B). Representative raw ${ }^{1} \mathrm{H}$ MR stimulated (Fig.

20 5C) and REST (Fig. 5D) spectra acquired during the same paradigm of stimulation were

21 acquired. The mean relative linewidth changes in NAA $\left(\Delta \mathrm{lw}_{\mathrm{NAA}}\right)$ were $-2.8 \pm 4 \%(\mathrm{n}=8)$ and

22 the mean relative $\mathrm{tCr}$ linewidth changes $\left(\Delta \mathrm{lw}_{\mathrm{tCr}}\right)$ were $-1.4 \pm 4 \%(\mathrm{n}=8)$. 


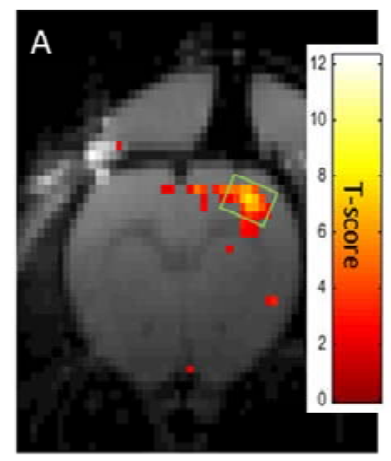

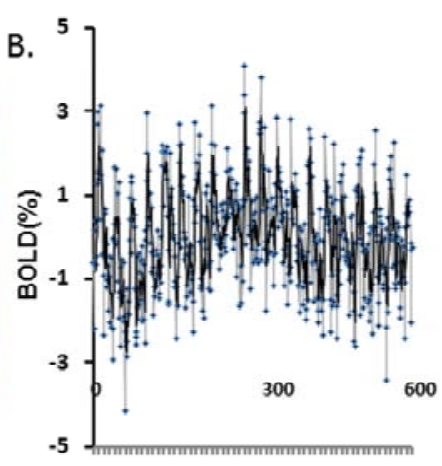

Time (s)

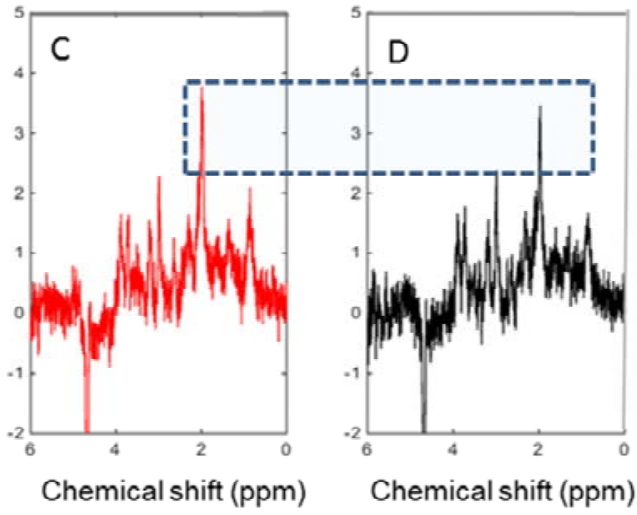

Chemical shift (ppm)

1 Figure 5: Characterization of BOLD effects on and tCr signals : A. Typical T-value BOLD map overlaid onto an anatomical image of the rat brain demonstrating activation in S1FL in a rat upon optogenetic stimulation. B. Typical BOLD timecourse during a 10s-ON-20s OFF paradigm of stimulation lasting 10 minutes. $C$ and $D$. Single rat ${ }^{1} H-M R$ spectra acquired in a $8 \mu \mathrm{l}$ voxel of interest covering the activated NAA area (as shown in Fig. 5A) during optogenetic stimulations (325 FIDs)(C, red) and resting periods (D, black). Boths spectra were reconstructed from FIDs acquired during the same paradigm of stimulation (5min-ON-5-min OFF, 45 minutes). The blue dotted rectangle serves to show the slightly increased amplitude of NAA in the stimulated spectrum compared to the resting spectrum.

10 For an improved characterization of the BOLD effects on ${ }^{1} \mathrm{H}$ MR spectra, water, NAA and tCr signals were measured over time during optogenetic stimulation paradigms ( $5 \mathrm{~min}$ ON-5min OFF). NAA peak height followed the optogenetic stimulation paradigm (Stimulation vs. REST vs. Stimulation: $8.4 \pm 6 \%$ vs. $3.2 \pm 4.3 \%$ (P >0.05) vs. $4.5 \pm 4.4 \%$; $\mathrm{P}<0.05$; Paired student t-test; Fig. 6A). The relative change of tCr peak height did not follow the paradigm of stimulation (Fig. 6B).

16 Unsuppressed water signals were also acquired during the paradigm of stimulation as a 17 function of time (n=5) (Fig. 6C).

A

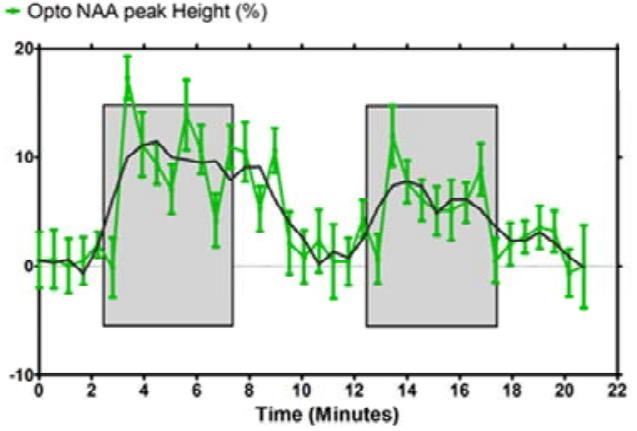

B

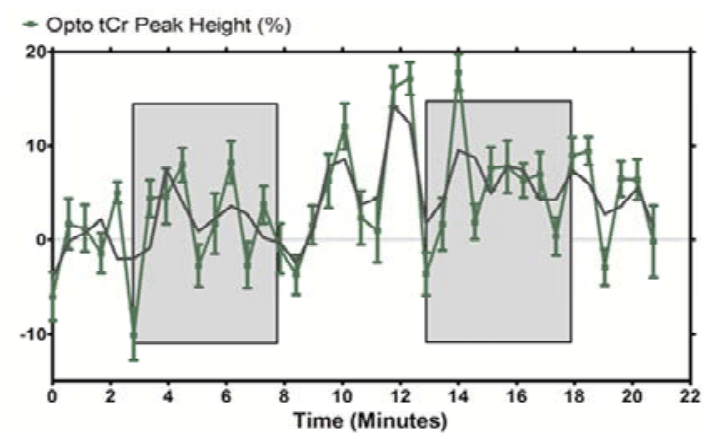


$\mathrm{C}$

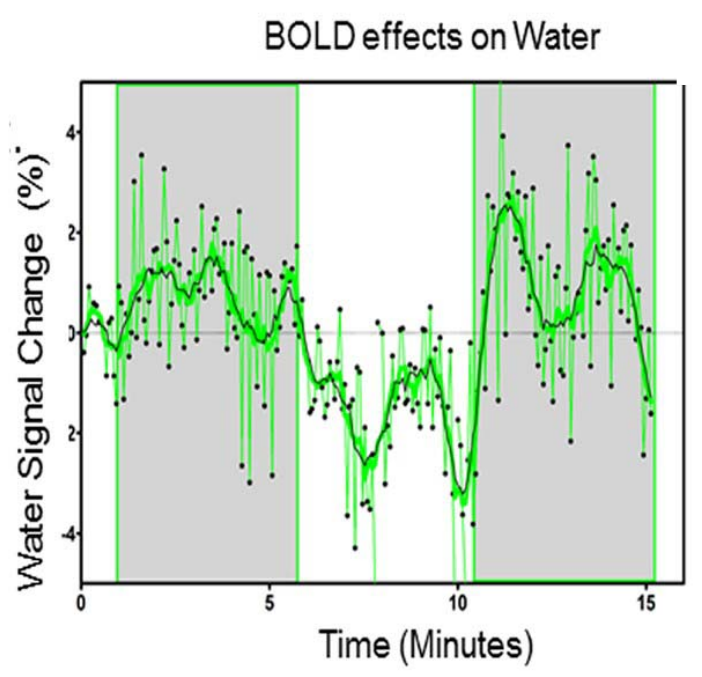

$\mathrm{D}$

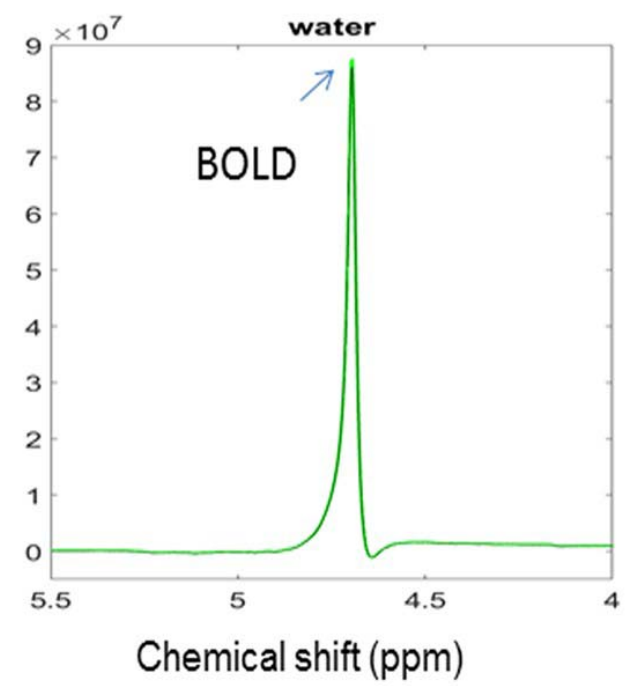

1 Figure 6: A. Quantification of NAA and B. tCr relative peak height changes $(\% \pm$ s.d) for 2 optogenetic stimulations Shaded areas indicate the periods of stimulation. Statistical 3 comparisons are described in the text. C. Quantification of water peak height changes (\%) for 4 optogenetic stimulations during the paradigm. Shaded areas indicate periods of stimulation. 5 D. The unsuppressed water peaks were temporally averaged for resting periods (black) and 6 stimulated periods (green) demonstrating a difference in amplitude (arrow) that can be 7 attributed to BOLD effects. Averaged water peaks were both used for further quantification of 8 metabolite concentrations.

Green laser stimulation induced significant increases in water peak heights over 5 minutes of

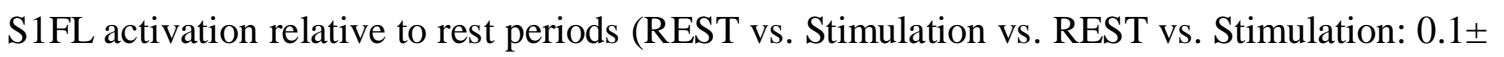
$0.7 \%$ vs. $0.8 \pm 1.2 \%(\mathrm{P}=0.013)$ vs $-1.6 \pm 1.4 \%(\mathrm{P}<0.001)$ vs. $1.1 \pm 1.5(\mathrm{P}<0.001)$, Student $\mathrm{t}-$ test; Fig.6C; mean \pm standard deviation). Stimulated and resting water peaks were averaged into single water peaks shown overlaid in Fig. 6D. The mean relative linewidth changes in water $\left(\Delta \mathrm{lw}_{\text {water }}\right)$ were $-0.5 \pm 0.8 \%$. Mean peak height and linewidth changes between REST and stimulation are summarized in Table 2. 
1 Table 2. In-vivo relative percent change of the height and linewidth of Water, NAA and

2 tCr peaks (optogenetic versus REST)

\begin{tabular}{cccccc} 
& $\begin{array}{c}\text { \% Height } \\
\text { Change }\end{array}$ & & \multicolumn{3}{c}{$\begin{array}{c}\% \\
\text { Linewidth } \\
\text { Change }\end{array}$} \\
\hline Water & NAA & tCr & Water & NAA & tCr \\
$1 \pm 1.0$ & $3.3 \pm 7$ & NA & $-0.5 \pm 0.8$ & $-2.8 \pm 4$ & $-1.4 \pm 4$
\end{tabular}

$4 \quad 3.3$ In-vivo quantification of metabolite concentration changes affected by BOLD effects

5 Population-averaged ${ }^{1} \mathrm{H}$ MR spectra $(\mathrm{n}=8)$ acquired during optogenetic stimulation and REST

6 are depicted in Fig.7a and $7 \mathbf{b}$ respectively. The REST spectrum was subtracted from the

7 stimulated spectrum resulting in a difference spectrum (Fig. 7c). The NAA peaks of the

8 stimulated and REST spectra were line-matched using a line broadening factor of $0.5 \mathrm{~Hz}$. The

$9 \mathrm{lb}$ factor was gradually increased in steps of $0.2 \mathrm{~Hz}$ up to $1 \mathrm{~Hz}$ by minimizing the residual

10 NAA difference peak (Inset). Each ${ }^{1} \mathrm{H}$ MR spectrum was individually fitted using LCModel.

11 Their subtraction resulted in a BOLD-corrected difference spectrum (Fig.7d). An identical

12 line-matching procedure was performed using $\mathrm{lb}=6 \mathrm{~Hz}$ corresponding to a $6 \%$ change in

13 NAA peak height during optogenetic stimulation. The $6 \mathrm{~Hz}$ line broadened spectrum was also

14 LCModel fitted. Metabolite concentrations quantified using LCModel and their changes are

15 summarized in Table 3. 


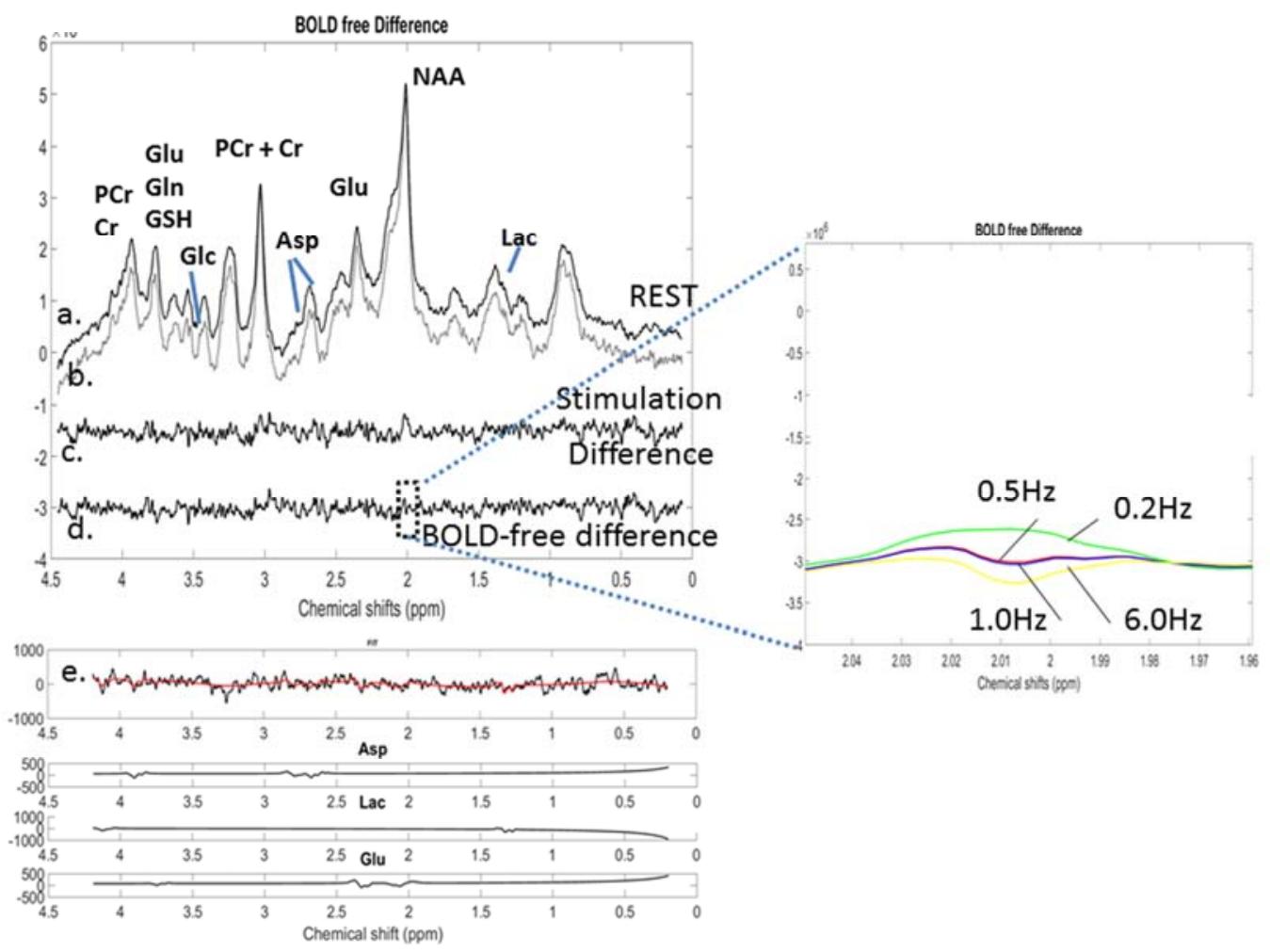

2 Figure 7: o-fMRS: Averaged (n=8) and labeled proton MR spectra for a. resting conditions and 3 for b. Photo-stimulation with green light. c: The REST spectrum was subtracted from the 4 stimulated spectrum prior to any correction for BOLD effects d. The BOLD free difference spectrum was obtained following application of a $0.5 \mathrm{~Hz}$ line broadening to minimize BOLD effects. e. LCModel fitting of the BOLD free difference spectrum allowed identification of the polarity of metabolic changes and retrieved Asp, Lac and Glu components included in the simulated basis set of the BOLD-free difference spectrum. Inset: NAA residuals for $l b=0.2 \mathrm{~Hz}$, $0.5 \mathrm{~Hz}, 1 \mathrm{~Hz}$ and $6 \mathrm{~Hz}$ resulting from the subtraction of the REST spectrum from line-broadened stimulated spectrum. The NAA residual was best minimized for $\mathrm{lb}=0.5 \mathrm{~Hz}$

Linewidth-matching techniques resulted in metabolite concentrations dropping by $-0.06 \pm$

$13 \mu \mathrm{mol} / \mathrm{g}$ on average for $\mathrm{lb}=0.5 \mathrm{~Hz}$ and $-0.18 \pm \mu \mathrm{mol} / \mathrm{g}$ for $\mathrm{lb}=6 \mathrm{~Hz}$ compared to uncorrected

14 concentrations. The number of metabolite concentrations changing significantly due to 15 stimulation differed with each analysis: waterBOLD vs STIMC (lb=0.5 Hz): 9 metabolites out 16 of $11 ; \mathrm{p}<0.01$; waterBOLD vs STIMC $(\mathrm{lb}=6 \mathrm{~Hz}): 4$ metabolites out of $11 ; \mathrm{p}<0.01$; STIMC $17(\mathrm{lb}=0.5 \mathrm{~Hz})$ vs STIMC $(\mathrm{lb}=6 \mathrm{~Hz}): 6$ metabolites out of $11 ; \mathrm{p}<0.01)$. 
1 Relative to STIM, water scaling with waterBOLD induced modest metabolic concentration

2 changes (below $\pm 0.3 \mu \mathrm{mol} / \mathrm{g}$ ) whereas the line-matching-techniques induced important

3 concentration changes for Asp and Glu concentrations $(\sim-1.0 \mu \mathrm{mol} / \mathrm{g})$. The changes in Glu

4 concentrations were in the range +0.4 to $+1.59 \mu \mathrm{mol} / \mathrm{g}$ for all $\mathrm{BOLD}$ corrections and

5 remained highly significant (Table 3). This was also the case for Asp concentrations ranging

6 from -1.16 to $-0.24 \mu \mathrm{mol} / \mathrm{g}$.

7 In an identical manner to the previous simulated data, the total BOLD effects measured in-

8 vivo can be calculated as the sum of the BOLD effects on water and the BOLD effects on

9 metabolites. As for the simulated data, BOLD effects on water were calculated as the

10 difference between metabolite concentrations quantified on the stimulated MR spectra with

11 waterREST and waterBOLD respectively. Assuming that the line-broadening correction leads

12 to BOLD-free metabolite concentrations, the BOLD effects on metabolites were calculated as

13 the difference between stimulated metabolite concentrations scaled with waterBOLD and

14 these BOLD-free metabolite concentrations. Thus, for NAA assumed as an intracellular

15 metabolite and a neuronal marker:

$16 \mathrm{BOLD}_{\mathrm{G}}(\mathrm{NAA})=\mathrm{BOLD}_{\text {water }}+\mathrm{BOLD}_{\text {metab }}=3.3 \%+2.91 \%=6.2 \%$ and $\Delta[\mathrm{NAA}]=-2.7 \%$

$$
=0.29+0.24=0.53 \mu \mathrm{mol} / \mathrm{g}
$$

18 For Glu, the same applies:

$\mathrm{BOLD}_{\mathrm{G}}(\mathrm{Glu})=\mathrm{BOLD}_{\text {water }}+\mathrm{BOLD}_{\text {metab }}=2.3 \%-1.93 \%=0.37 \%$ and $\Delta[\mathrm{Glu}]=7.7 \%$

$$
==0.16-0.14=0.02 \mu \mathrm{mol} / \mathrm{g}
$$

21 Glu also represents a neuronal marker but is also present in the extracellular space during 22 synaptic release [24]. Exchanges between intracellular and extracellular spaces may explain 23 the negative effect on metabolites and a lesser effect of global BOLD $\left(\mathrm{BOLD}_{\mathrm{G}}\right)$. In the same 24 manner, BOLD effects on a glial marker such as Ins, can be separated. The negative 25 contribution of BOLD effects on water may be attributed to changes in water dynamics within 26 astrocytes [25]. 
$1 \mathrm{BOLD}_{\mathrm{G}}(\mathrm{Ins})=\mathrm{BOLD}_{\text {water }}+\mathrm{BOLD}_{\text {metab }}=-4.7 \%+7.85 \%=3.15 \%$ and $\Delta[$ Ins $]=21.6 \%$

2

$$
=-0.18+0.28=0.1 \mu \mathrm{mol} / \mathrm{g}
$$

3 BOLD effects can thus substantially contaminate metabolite concentrations.

\subsection{Characterization and quantification with BOLD-free difference spectrum}

5 BOLD-free difference spectra were fitted using LCModel and a simulated basis set including Asp,

6 Glu, Glc and Lac (Fig. 7e). The obtained concentration changes for Asp and Glu presented in Table 4

7 were in agreement with the group analysis performed between averaged STIM and REST spectra

8 (Table 3). A negative Lac peak was also determined. Upon visual inspection of the overlaid

9 optogenetic and REST spectra (Fig. 8), positive peaks were observed for Glu (at $2.35 \mathrm{ppm}$ ) as well as

10 negative peaks at $3.75 \mathrm{ppm}$. A negative Asp peak was also identified (2.77ppm). Moreover, positive

11 GSH peaks were also observed (at 2.92/2.97 and $3.78 \mathrm{ppm}$ ). At $1.32 \mathrm{ppm}$, the stimulated Lac peak was

12 under the non-stimulated one resulting in a negative Lac peak in the BOLD-free difference spectrum.

13 In addition, at $3.5 \mathrm{ppm}$, the peak corresponding to Glc disappeared upon optogenetic stimulation,

14 resulting in a negative peak. The quantitative outcome of the LCModel fit to the BOLD-free

15 difference spectrum is reported in Table 4 although noise levels remained important impeding an

16 appropriate and precise quantification of changes using LCModel. 


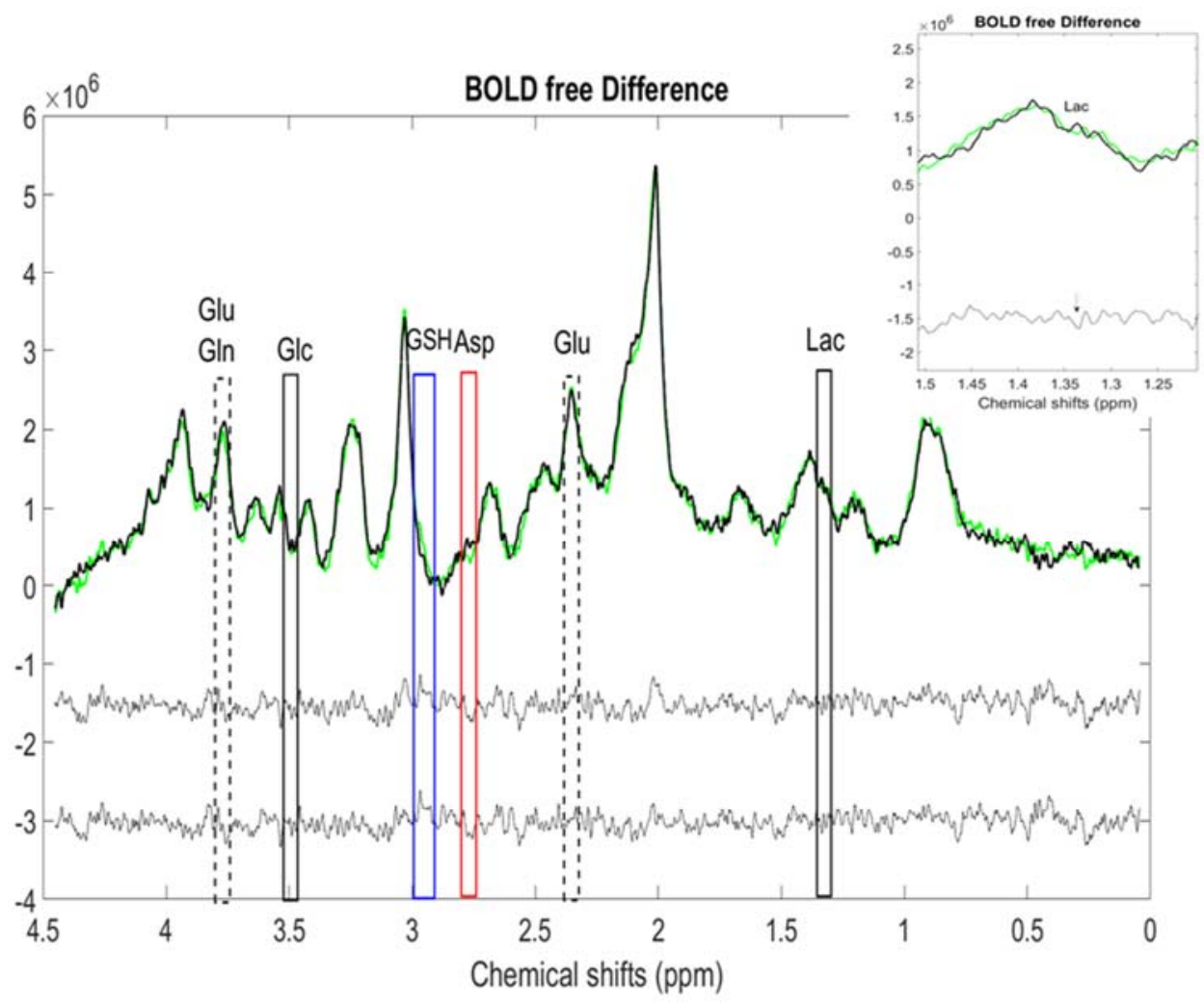

1

2 Figure 8: Qualitative observation of amplitude spectral changes: Optogenetically stimulated 3 and REST spectra ( $n=8)$ were overlaid allowing enhanced observation of metabolite changes. 4 Overlaid peaks ascribed to lactate (Lac (1.32ppm); plain black rectangle), glucose (Glc (3.5 $5 \mathrm{ppm})$ ), glutamate (Glu (2.12, 2.35 and $3.75 \mathrm{ppm}$; dotted rectangles)), aspartate (Asp (2.77 ppm); 6 red rectangle) as well as glutathione (GSH $(2.92,2.97$ and $3.78 \mathrm{ppm})$; blue rectangle) were 7 identified. Inset: The arrow indicates a peak of Lac at 1.32 ppm at REST but not during green 8 laser stimulation: amplitude differences lead to a negative peak in the BOLD-free difference spectrum (arrow). 
1 Table 3:Quantification of in-vivo metabolite concentrations: Baseline, Stimulated and BOLD corrected metabolite concentrations were obtained through

2 adjustments of MR spectra with LCModel. Stimulated MR spectra were quantified using waterREST and WaterBOLD respectively. The optimized line

3 broadening factor (see Fig.7) was obtained for $\mathrm{lb}=0.5 \mathrm{~Hz}$. An erroneous $\mathrm{lb}=6 \mathrm{~Hz}$ was also used. A group analysis was performed and one way ANOVAs

4 were performed to evaluate the significance of metabolite concentration changes. Bonferroni post-hoc tests were used for multiple comparisons. * p<

$5 \quad 0.05 ; * * p<0.01 ; * * * p<0.001$

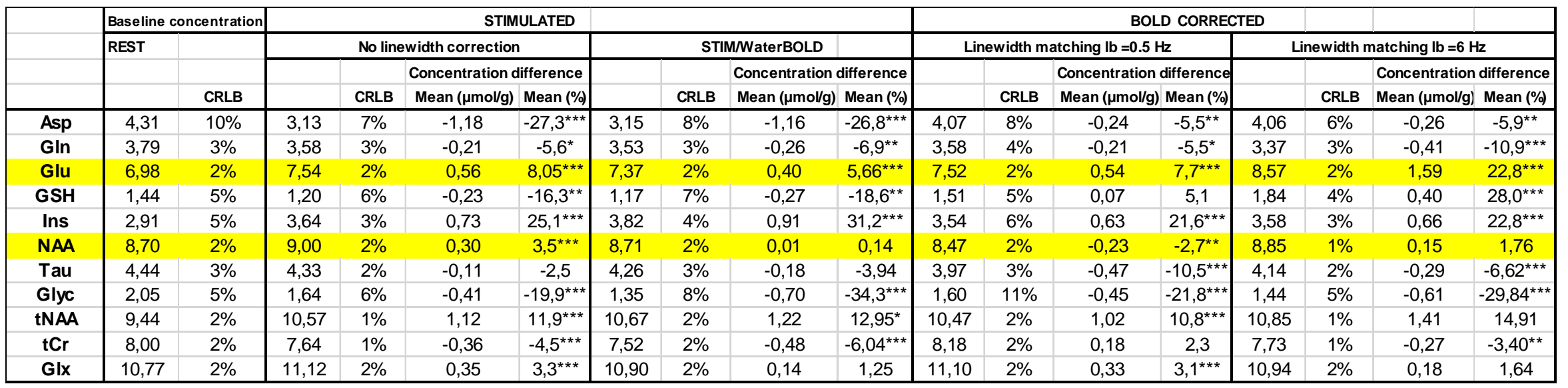


1 Table4: Quantification of metabolite changes with LCModel

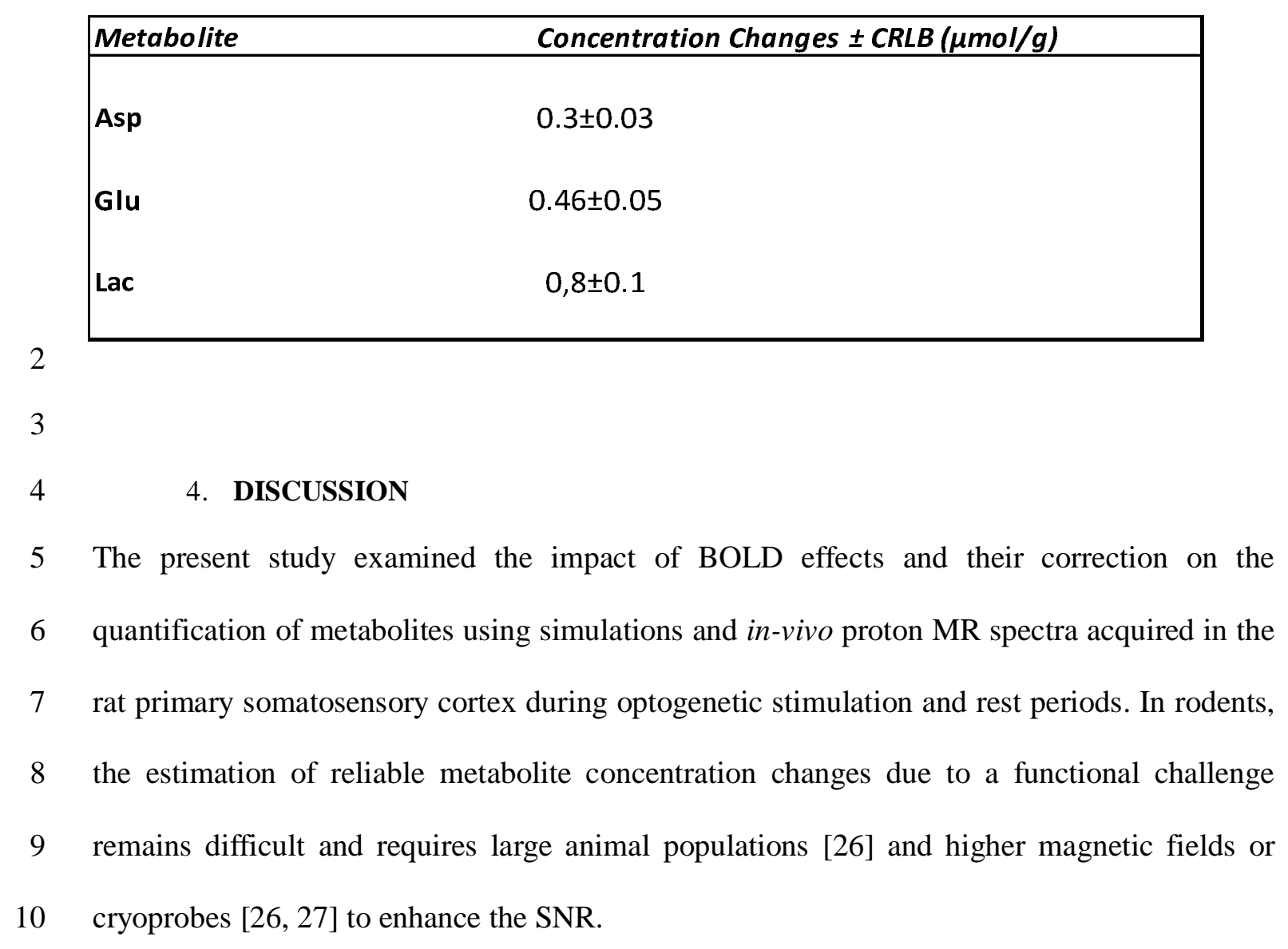

\section{$11 \quad$ 4.1 The line-matching procedure removes BOLD effects}

12 Removal of BOLD effects in ${ }^{1} \mathrm{H}$-fMRS allows for the estimation of metabolite concentration

13 changes solely attributed to neurochemical changes as a consequence of brain activity

$14[1,5,6,8,10,11,12,13,14,28]$. BOLD effects generate small changes in metabolite

15 concentrations that are not necessarily of identical magnitude for all metabolites [8, 29].

16 When not corrected, metabolic concentration changes may appear erroneously significant

17 [12]. The appropriate line broadening correction for BOLD effects is based on the value that

18 best minimizes the residuals of $\mathrm{tCr}$ and NAA peaks resulting from the subtraction of the

19 population-averaged stimulated and REST proton MR spectra. This procedure strongly relies

20 on an optimized phasing of both the stimulated and REST spectra to limit potential frequency

21 drifts during the subtraction. Low SNR levels in small VOIs of the rat cortex further 
1 complicate the subtraction methodology. A more direct quantification of BOLD- free

2 metabolite concentration changes with LCmodel could simplify ${ }^{1} \mathrm{H}$-fMRS data analysis and 3 interpretation.

4 Recent studies reported that LCModel analysis of short-TE data was highly sensitive to noise 5 and spectral linewidth variations [30]. Notably, line broadening of original data analyzed with

6 LCModel induced substantial metabolite quantification changes. Therefore, the reliability of 7 metabolite quantification following linewidth matching for BOLD correction can be 8 questioned. In the present study, the simulation of BOLD effects demonstrated that the line9 matching procedure is an effective method to remove them with a quantification error of less 10 than $1 \%( \pm 0.05 \mu \mathrm{mol} / \mathrm{g})$. However, if the line broadening factor is different from the line11 narrowing factor induced by BOLD effects, up to a $5 \%( \pm 0.3 \mu \mathrm{mol} / \mathrm{g})$ error in quantification 12 may be obtained, which is substantial when absolute metabolic changes as low as $0.2 \mu \mathrm{mol} / \mathrm{g}$ 13 are expected $[4,5,6]$.

\section{$14 \quad 4.2$ Water Scaling}

15 For the absolute quantification of metabolites with LCModel, the unsuppressed water peak 16 acquired in the same VOI as the ${ }^{1} \mathrm{H}-\mathrm{MR}$ spectrum serves for scaling and for eddy -current 17 correction. During stimulation, the water peak undergoes a line-narrowing effect due to the BOLD effects. As LCMOdel [9] considers resonance areas, the water line-narrowing due to $19 \mathrm{~T}_{2} *$-induced effects should not affect metabolite quantification. The simulations used in the 20 present study showed that this was not the case. Using waterBOLD for scaling, the error 21 induced on metabolite concentrations was lower than with waterREST. The quantification of 22 metabolite concentrations on a proton MR spectrum with BOLD simulated contamination 23 suggested that compensatory effects take place when using stimulated water (waterBOLD).

24 This finding led us to postulate that metabolite concentrations quantified using waterBOLD 25 are affected by BOLD effects on metabolites only whereas metabolite concentrations 26 quantified using waterREST contain the cumulative BOLD effects on metabolites and water. 
1 In single-voxel MR spectroscopy, lineshapes can be distorted by B0 field inhomogeneities and

2 eddy currents. Metabolite fitting models using water reference lines to compensate for B0

3 field inhomogeneity have also been used in combination with LCModel [9] for quantification

4 purposes. Notably, increased accuracy and stability of spectral quantification were found

5 using the reference lineshapes from the water signal [17]. In the present study, the use of

6 waterBOLD for scaling, partially corrected for the change in spectral linewidth occasioned by

$7 \quad$ BOLD effects.

\section{$8 \quad 4.3$ Compartmentation of BOLD effects}

9 BOLD responses measured by fMRI or fMRS on water represent the $\mathrm{T}_{2}$-induced effects 10 encompassing both intravascular and extravascular compartments. Since BOLD fMRI 11 provides an indirect measure of neuronal activity and relies on neurovascular coupling 12 mechanisms, diffusion fMRI (dfMRI) [31] has been proposed as a possible method to 13 disentangle the vascular and extravascular components of BOLD. dfMRI relies on 14 microstructural changes driven by neural activity such as cell swelling to induce changes in 15 the diffusivity of water molecules.

16 Another technique independent of neurovascular coupling and taking advantage of the 17 specific distribution of metabolites within different tissue compartments is MRS. Neuronal 18 compartments and other extravascular and intravascular compartments may be differentially 19 affected by $\mathrm{T}_{2}{ }^{*}$-induced effects. For example, $\mathrm{T}_{2}{ }^{*}$-induced effects measured with ${ }^{1} \mathrm{H}$-fMRS 20 on the neuronal markers NAA and Glu and on the glial marker Ins may represent the effects 21 on the neuronal compartment and the glial compartment [7]. In the human visual cortex at 4T, 22 the changes in linewidth and amplitude were similar for water and metabolites [7], which was 23 not the case in the present study at 9.4T. Thus, the linewidth and amplitude changes of the 24 spectral lines assessed with ${ }^{1} \mathrm{H}$-fMRS could represent specific markers of the BOLD effects in 25 extravascular compartments.

26 Older studies revealed that following injections of the paramagnetic agent (Dy-TTHA) ${ }^{3-}$ and a 
1 vasodilator, the water peak in the rat brain divided into 3 peaks representing intracellular

2 water, extravascular-extracellular water and intravascular water. The distribution of $\mathrm{Na}^{+}$ions

3 in the 3 different compartments was also shown [32]. When blocking the sodium pump with

4 ouabain, a slight increase of the intracellular water peak signal was noticed corresponding to

5 an increase of the intracellular volume. The intravascular and extravascular-extracellular

6 volumes became almost negligible.

7 By extrapolation, the BOLD effects assessed by MRS on a spectral water peak could be 8 compared to the changes seen in [32]. Moreover, upon depolarization induced by a stimulus, a

$9 \mathrm{Na}^{+}$gradient towards the intracellular milieu occurs. This analogy was used in the past with

10 diffusion magnetic resonance imaging (dMRI) to verify that the dynamic swelling of neurons

11 due to neuronal activation, induced a decrease of the apparent diffusion coefficient (ADC)

12 [33]. Potential changes in physical environments inducing ADC changes would also affect $\mathrm{T}_{2}$.

13 Since $\mathrm{T}_{2}$ changes also occur as a result of focal cortical activation, cell swelling or 14 macromolecular changes may also be envisaged in the present study. Other groups also 15 demonstrated important BOLD effects on the neuronal compartment, which origin was also 16 attributed to cell swelling or macromolecular changes [7, 34]. At 9.4T, a negligible effect of 17 intravascular BOLD was revealed [34]. If cell swelling due to neural activation is assumed, 18 then vascular BOLD effects can also be neglected. Changes in water and NAA signals during 19 optogenetic stimulation could therefore be related to an increase of the intracellular volume.

20 Diffusion functional MRS (dfMRS) was also proposed as a new tool to separate the changes 21 in properties of neuronal spaces from the hemodynamic response during neuronal activity 22 [35]. Despite the potential of both diffusion techniques, many measurements remain to be 23 performed to ensure their validation as probes for the direct measurement of neural activity.

24 Besides, both methods require additional work to validate the underlying mechanisms and 25 both are suspected to remain contaminated by residual BOLD signals. The present work 26 participates in the effort to better identify genuine neural activity. A simple method was 
1 proposed to separate water and intracellular BOLD effects and will need to be validated.

\section{$2 \quad 4.3$ In-vivo evaluation of BOLD effects}

3 Evaluation of the in-vivo water and NAA peak amplitude changes as a function of time

4 followed the stimulation paradigm during periods of photo-stimulation and periods of REST,

5 mimicking BOLD changes usually seen in BOLD fMRI time courses. These findings suggest

6 that estimating water and NAA peak height changes represents an acceptable means of

7 identifying spectral BOLD effects in the rat cortex. On the contrary, changes in tCr peak

8 amplitudes as a function of time did not follow the paradigm of stimulation. In a very recent

9 study conducted at $9.4 \mathrm{~T}$ in the human brain, $\mathrm{tCr}$ concentrations did not follow the visual

10 stimulation paradigm, while Cr concentrations did [36]. These results confirm that BOLD

11 effects may be counterbalanced by the conversion of $\mathrm{PCr}$ into $\mathrm{Cr}$ and vice versa as hinted by

12 the absence of observable $\mathrm{T}_{2}$ changes in other studies [34]. In rodents, Medetomidine sedation

13 may also be responsible for some modulation of metabolite levels [37].

14 In-vivo, stimulated spectra needed to be corrected for the BOLD effect without impacting the

15 potential metabolite concentration changes due to optogenetic stimulation. Overall, the

16 corrected amplitude of changes in metabolite concentrations upon stimulation as well as the

17 direction (positive or negative) of these changes was in agreement with previous studies in

18 rodents $[13,14,15,16,26]$ for all the different methods. The best agreement with previous

19 literature values on functional metabolic concentration changes was found for the line-

20 matching procedure $[13,14,15,26]$ with $\mathrm{lb}=0.5 \mathrm{~Hz}$. Using the line-matching procedure with

$21 \mathrm{lb}=6 \mathrm{~Hz}$, changes in Asp and Glu concentrations were above $-1 \mu \mathrm{mol} / \mathrm{g}$ (Table 3), which were

22 largely overestimated compared to previous reports [13,14,15]. Moreover, the linewidth-

23 matching correction with $\mathrm{lb}=6 \mathrm{~Hz}$ demonstrated to be erroneous (Fig. 7). Given these results,

24 the line-matching procedure using $\mathrm{lb}=0.5 \mathrm{~Hz}$ appears the most suitable for correcting BOLD

25 effects without affecting metabolic concentration changes due to optogenetic stimulation in

26 the present study. 


\section{$1 \quad 4.4$ BOLD-free difference spectra}

2 Here, findings with BOLD free difference spectra were considered to remain only indicative

3 of the polarity of changes due to low SNR. Nevertheless, direct LCModel fitting led to

4 reasonable quantitative estimates for Glu and Asp concentration changes (Table 4) compared

5 to changes reported in Table 3. The CRLB for Lac were $40 \%$ and thus were not reported. .

6 Observations were qualitatively performed by visual inspection of the overlaid linewidth-

7 corrected $(\mathrm{lb}=0.5 \mathrm{~Hz})$ stimulated and REST spectra. Subtraction of the resting spectrum from

8 the spectrum acquired during photo-stimulation and corrected for BOLD effects resulted in

9 small positive and negative peaks representing stimulus-related changes in several

10 metabolites. Positive (Glu, GSH, Lac) and negative (Asp, Lac) peaks representing metabolite

11 concentration changes were identified at specific chemical shift positions confirming

12 quantitative findings using LCModel fittings reported in Table 3.

\section{CONCLUSION}

15 In conclusion, BOLD effects can be quantified using ${ }^{1} \mathrm{H}$-fMRS in the rat cortex using ${ }^{1} \mathrm{H}$ fMRS. Linewidth-matching techniques remain the most reliable correction for eliminating

17 false positive errors in metabolite concentration estimates provided the extent of $\mathrm{T}_{2}$-induced effects is characterized for the precise determination of the line broadening factor. The ensuing calculation of a BOLD-corrected difference spectrum should then be more

20 straightforward and more specific. Alternatively, BOLD-related spectral changes may be 21 useful to further identify differences between cortical extravascular compartments upon 22 activation. As pointed out recently (38), novel $\mathrm{T}_{2} *$ methods are needed to better correct for 23 BOLD effects in ${ }^{1} \mathrm{H}-\mathrm{fMRS}$. At the same time, a better characterization of BOLD effects 24 independent of neurovascular coupling may be useful for an improved assessment of direct 25 neuronal activity. 


\section{References}

2 [1] Moreno A, Jego P, dela Cruz F,Canals S. Neurophysiological, metabolic and cellular compartments

3 that drive neurovascular coupling and neuroimaging signals. Front. In Neuroenergetics. 2013; 5:1-7.

4 [2] Logothetis N, Pauls J, Augath M, Trinath T, Oldermann A. Neurophysiological investigation of the 5 basis of fMRI signal. Nature.2001. 412, 150-157.

6 [3] Mangia S, Giove F, Tkac I, Logothetis NK, Henry PG, Olman CA, Maraviglia B, Di Salle F,

7 Ugurbil K. Metabolic and hemodynamic events after changes in neural activity: current hypotheses, 8 theoretical predictions and in-vivo NMR findings. J Cereb Blood Flow Metab. 2009 ;29:441-63.

9 [4] Mangia S, Tkác I, Logothetis NK, Gruetter R, Van de Moortele PF, Uğurbil K. Dynamics of 10 lactate concentration and blood oxygen level-dependent effect in the human visual cortex during 11 repeated identical stimuli. J Neurosci Res. 2007; 85(15):3340-6.

12 [5] Mangia S, Tkác I, Gruetter R, Van de Moortele PF, Maraviglia B, Uğurbil K. Sustained 13 neuronal activation raises oxidative metabolism to a new steady-state level: evidence from $1 \mathrm{H}$ 14 NMR spectroscopy in the human visual cortex. J Cereb Blood Flow Metab. 2007 ;27(5):1055-63.

15 [6] Schaller B, Mekle R, Xin L, Kunz N, Gruetter R (2013) Net increase of lactate and glutamate 16 concentration in activated human visual cortex detected with magnetic resonance spectroscopy at 7 17 tesla. J Neurosci Res 91(8):1076-1083. http://dx.doi.org/10.1002/ jnr.23194.

18 [7] Zhu XH, Chen W. Observed BOLD effects on cerebral metabolite resonances in human visual 19 cortex during visual stimulation: a functional (1)H MRS study at 4 T. Magn Reson Med. $20 \quad 2001 ; 46(5): 841-7$

21 [8] Bednařík P, Tkáč I, Giove F, Eberly LE, Deelchand DK, Barreto FR, Mangia S. Neurochemical 22 responses to chromatic and achromatic stimuli in the human visual cortex.J.Cereb .Blood Flow Metab. 23 2018;38(2):347-359. doi: 10.1177/0271678X17695291.

24 [9] Provencher, SW (1993) Estimation of metabolite concentrations from localized in vivo proton 25 NMR spectra. Magn Reson Med 30:672-9

26 [10] Schaller B, Xin L, O’Brien K, Magill AW, Gruetter R (2014) Are glutamate and lactate

27 increases ubiquitous to physiological activation? A (1)H functional MR spectroscopy study during 28 motor activation in human brain at 7Tesla. Neuroimage 93(Pt 1):138-145. 29 http://dx.doi.org/10.1016/j.neuroimage.2014.02.016.

30 [11] Lin Y, Stephenson MC, Xin L, Napolitano A, Morris PG.. Investigating the metabolic changes due 31 to visual stimulation using functional proton magnetic resonance spectroscopy at $7 \square$ T.J Cereb Blood 32 Flow Metab. 2012 Aug;32(8):1484-95. doi: 10.1038/jcbfm.2012.33.

33 [12] Bednařík P, Tkáč I, Giove F, DiNuzzo M, Deelchand DK, Emir UE, Eberly LE, Mangia S. 34 Neurochemical and BOLD responses during neuronal activation measured in the human visual cortex 35 at 7 Tesla. J. Cereb Blood Flow Metab. 2015 31;35(4):601-10. doi: 10.1038/jcbfm.2014.233. 
1 [13] Just N, Xin L, Frenkel H, Gruetter R. Characterization of sustained BOLD activation in the rat 2 barrel cortex and neurochemical consequences. Neuroimage.2013;74:343-51. doi: 3 10.1016/j.neuroimage.2013.02.042.

5

6

[14] Just N, Sonnay S. Investigating the Role of Glutamate and GABA in the Modulation of Transthalamic Activity: A Combined fMRI-fMRS Study. Front Physiol. 2017; 8:30. doi: 10.3389/fphys.2017.00030. eCollection 2017.

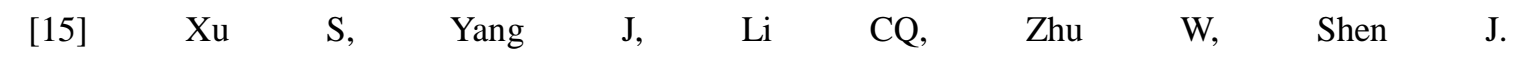
Metabolic alterations in focally activated primary somatosensory cortex of alpha-chloraloseanesthetized rats measured by 1 H MRS at 11.7 T. Neuroimage. 2005; 28(2):401-9.

[16] Just N, Faber C. Probing activation-induced neurochemical changes using optogenetics combined with functional magnetic resonance spectroscopy: a feasibility study in the rat primary somatosensory cortex. J Neurochem. 2019 Aug;150(4):402-419. doi: 10.1111/jnc.14799.

[17] Hong D, van Asten JJA, Rankouhi SR, Thielen JW, Norris DG. Effect of linewidth on estimation of metabolic concentration when using water lineshape spectral model fitting for single voxel proton spectroscopy at 7 T. J Magn Reson. 2019 Jul;304:53-61. doi: 10.1016/j.jmr.2019.05.002.

[18] Zöllner HJ, Považan M, Hui SCN, Tapper S, Edden RAE, Oeltzschner Comparison of different linear-combination modeling algorithms for short-TE proton spectra. NMR Biomed. 2021 Apr;34(4):e4482. doi: 10.1002/nbm.4482.

[19] Yizhar O, Fenno LE, Davidson TJ, Mogri M, Deisseroth K.Optogenetics in neural systems.Neuron. 2011 ;71(1):9-34.doi:10.1016/j.neuron.2011.06.004.

[20] Christie IN, Wells JA, Southern P, Marina N, Kasparov S, Gourine AV, Lythgoe MF fMRI response to blue light delivery in the naïve brain: implications for combined optogenetic fMRI studies. Neuroimage. 2013; 66:634-41. doi: 10.1016/j.neuroimage.2012.10.074

[21] Tkác I, Starcuk Z, Choi IY, Gruetter R.In vivo 1H NMR spectroscopy of rat brain at $1 \mathrm{~ms}$ echo time.Magn Reson Med. 1999 ;41(4):649-56.

[22] Peters, A.M., Brookes, M.J., Hoogenraad, F.G., Gowland, P.A., Francis, S.T., Morris, P.G., Bowtell, R., 2007. T-2* measurements in human brain at 1.5, 3 and 7 T. Magn. Reson. Imaging 25 , $748-753$

[23] van der Zwaag W, Francis S, Head K, Peters A, Gowland P, Morris P, Bowtell R.fMRI at 1.5, 3 and 7 T: characterising BOLD signal changes.Neuroimage. 2009;47(4):1425-34. doi: 10.1016/j.neuroimage.2009.05.015.

[24] Moussawi K, Riegel A, Nair S, Kalivas PW. Extracellular glutamate: functional compartments operate in different concentration ranges. Front Syst Neurosci. 2011 Nov 24;5:94. doi: 10.3389/fnsys.2011.00094 
1 [25] Borrachero-Conejo AI, Adams WR, Saracino E, Mola MG, Wang M, Posati T, Formaggio F, De

2 Bellis M, Frigeri A, Caprini M, Hutchinson MR, Muccini M, Zamboni R, Nicchia GP, Mahadevan-

3 Jansen A, Benfenati V. Stimulation of water and calcium dynamics in astrocytes with pulsed infrared

4 light. FASEB J. 2020 May;34(5):6539-6553. doi: 10.1096/fj.201903049R.

5 [26] Sonnay S, Duarte JMN, Just N. Lactate and glutamate dynamics during prolonged stimulation of

6 the rat barrel cortex suggest adaptation of cerebral glucose and oxygen metabolism.Neuroscience.

7 2017;346:337-348. doi:10.1016/j.neuroscience.2017.01.034.

8 [27] Iordanova B, Vazquez AL, Poplawsky AJ, Fukuda M, Kim SG. Neural and hemodynamic

9 responses to optogenetic and sensory stimulation in the rat somatosensory cortex. J Cereb Blood Flow

10 Metab. 2015 ;35(6):922-32. doi: 10.1038/jcbfm.2015.10.

11 [28] Just N. Proton functional magnetic resonance spectroscopy in rodents. NMR Biomed. 2021

12 May;34(5):e4254. doi: 10.1002/nbm.4254.

13 [29] Mangia S, Tkác I, Gruetter R, Van De Moortele PF, Giove F, Maraviglia B, Uğurbil K.Sensitivity

14 of single-voxel 1H-MRS in investigating the metabolism of the activated human visual cortex at 7

15 T.Magn Reson Imaging. 2006 May;24(4):343-8.

16 [30] Zhang Y, Shen J. Effects of noise and linewidth on in vivo analysis of glutamate at $3 \mathrm{~T}$. J Magn

17 Reson . 2020 May;314:106732. doi: 10.1016/j.jmr.2020.106732.

18 [31] Le Bihan D, Urayama S, Aso T, Hanakawa T, Fukuyama H. Direct and fast detection of neuronal 19 activation in the human brain with diffusion MRI.

20 Proc Natl Acad Sci U S A. 2006 May 23;103(21):8263-8. doi: 10.1073/pnas.0600644103.

21 [32] Naritomi H, Kanashiro M, Sasaki M, Kuribayashi Y, Sawada T. In vivo measurements of intra-

22 and extracellular $\mathrm{Na}+$ and water in the brain and muscle by nuclear magnetic resonance spectroscopy

23 with shift reagent. Biophys J. 1987 Oct;52(4):611-6. doi: 10.1016/S0006-3495(87)83251-4.

24 [33] Radecki G, Nargeot R, Jelescu IO, Le Bihan D, Ciobanu L. Functional magnetic resonance 25 microscopy at single-cell resolution in Aplysia californica. Proc Natl Acad Sci U S A. 2014 Jun 26 10;111(23):8667-72. doi: 10.1073/pnas.1403739111.

27 [34] Lei H, Zhang Y, Zhu XH, Chen W. Changes in the proton T2 relaxation times of cerebral water 28 and metabolites during forebrain ischemia in rat at 9.4 T. Magn Reson Med. 2003;49(6):979-84.

29 [35] Branzoli F, Techawiboonwong A, Kan H, Webb A, Ronen I. Functional diffusion-weighted 30 magnetic resonance spectroscopy of the human primary visual cortex at $7 \mathrm{~T}$.

31 Magn Reson Med. 2013 Feb;69(2):303-9. doi: 10.1002/mrm.24542.

32 [36] Dorst $\mathbf{J}$ et al. Simultaneous detection of metabolite concentration changes, water BOLD signal 33 and $\mathrm{pH}$ changes during visual stimulation in the human brain at 9.4 T. Proceedings of the International 34 Society of Magnetic Resonance in Medicine 2021. 339

35 [37] Boretius S, Tammer R, Michaelis T, Brockmöller J, Frahm J. Halogenated volatile anesthetics 36 alter brain metabolism as revealed by proton magnetic resonance spectroscopy of mice in 37 vivo.Neuroimage. $2013 ; 69: 244-55$. doi: 10.1016/j.neuroimage.2012.12.020 
1 [38] C Ligneul, FF Fernandes, N Shemesh. High temporal resolution functional Magnetic 2 Resonance Spectroscopy in the mouse upon visual stimulation: Metabolic changes upon 3 mouse visual stimulation detected using fMRS NeuroImage, $117973 ; 2021$

4

\section{6.Acknowlegements}

6 The author would like to thank the Translational Research Institute Center for providing animals. All

7 fMRS Matlab routines were adapted from previous routines developed in the Centre d'Imagerie

8 Biomédicale (Lausanne, Switzerland) by the first author.

9

10 Conflict of Interest: No conflict of interest.

11 